\title{
PILOT IN-TRAIL PROCEDURE VALIDATION SIMULATION STUDY
}

\author{
Frank J.L. Bussink ${ }^{1}$ \\ National Institute of Aerospace \\ Jennifer L. Murdoch², James P. Chamberlain ${ }^{3}$, Ryan Chartrand ${ }^{4}$, and Kenneth M. Jones ${ }^{5}$ \\ NASA Langley Research Center
}

A Human-In-The-Loop experiment was conducted at the National Aeronautics and Space Administration (NASA) Langley Research Center (LaRC) to investigate the viability of the In-Trail Procedure (ITP) concept from a flight crew perspective, by placing participating airline pilots in a simulated oceanic flight environment. The test subject pilots used new onboard avionics equipment that provided improved information about nearby traffic and enabled them, when specific criteria were met, to request an ITP flight level change referencing one or two nearby aircraft that might otherwise block the flight level change. The subject pilots' subjective assessments of ITP validity and acceptability were measured via questionnaires and discussions, and their objective performance in appropriately selecting, requesting, and performing ITP flight level changes was evaluated for each simulated flight scenario. Objective performance and subjective workload assessment data from the experiment's test conditions were analyzed for statistical and operational significance and are reported in the paper. Based on these results, suggestions are made to further improve the ITP.

\section{Introduction}

Aircraft in oceanic and remote non-radar airspace frequently fly for extended periods of time in the same direction and along a limited number of predefined flight paths. Since there is no radar surveillance and only limited direct communications available, controllers use procedural separation rules to ensure that aircraft remain separated. Procedural separation typically requires much greater time or distance between aircraft than when radar surveillance and direct communications are available. This limits the number of aircraft on a given track at a given altitude or flight level (FL).

Furthermore, an aircraft's most fuel-efficient altitude is lower during the early segments of a flight, when the aircraft has a heavy fuel load, and higher during the later segments when much of the fuel has been consumed; so operational efficiency is generally enhanced by climbing to a higher flight level one or more times during a long flight segment. However, in many situations the standard longitudinal separation interval does not exist at neighboring, or intervening, flight levels but may exist at a succeeding flight level. An aircraft desiring a flight level change to such a succeeding flight level would therefore be prevented or "blocked" from making the climb or descent through the intervening flight level(s) (Figure 1).

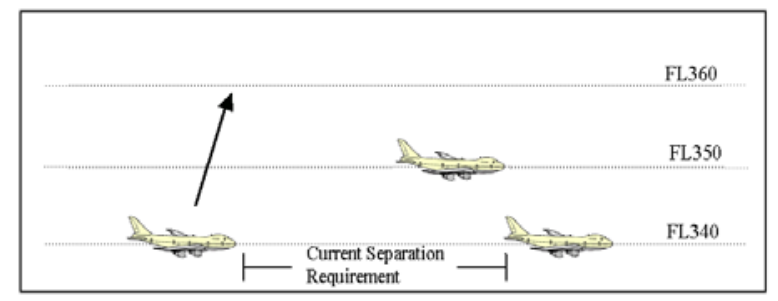

Figure 1. Aircraft at Flight Level 340 (FL340) desires a climb to FL360

A new In-Trail Procedure (ITP) was developed to enable climbs and descents through normally blocked flight levels, providing a safe and practical method for Air Traffic Service Providers (ATSP) to approve, and flight crews to conduct, such operations. This procedure was developed under the Enhanced Oceanic Operations (EOO) research element as part of the NASA Next Generation Air Transportation

\footnotetext{
${ }^{1}$ Frank J.L. Bussink - Mailing Address: Mail Stop 156A, NASA Langley Research Center, Hampton, VA 23681; | Email Address: <fjlbussink@gmail.com>; Telephone Number: 757-864-8304.

${ }^{2}$ Jennifer L. Murdoch - Mailing Address: Mail Stop 156A, NASA Langley Research Center, Hampton, VA 23681; Email Address: <Jennifer.L.Murdoch@nasa.gov>; Telephone Number: 757-864-8304.

${ }^{3}$ James P. Chamberlain - Mailing Address: Mail Stop 156A, NASA Langley Research Center, Hampton, VA 23681; Email Address: <James.P.Chamberlain@nasa.gov>; Telephone Number: 757-864-2147.

${ }^{4}$ Ryan C. Chartrand - Mailing Address: Mail Stop 156A, NASA Langley Research Center, Hampton, VA 23681; Email Address: <Ryan.C.Chartrand@nasa.gov>; Telephone Number: 757-864-2214.

${ }^{5}$ Kenneth M. Jones - Mailing Address: Mail Stop 156A, NASA Langley Research Center, Hampton, VA 23681;

Email Address: <Kenneth.M.Jones@nasa.gov>; Telephone Number: 757-864-5013.
} 
System (NextGen) Air Traffic Management (ATM) Airspace Project. EOO's objective was to develop methodologies, concepts, and procedures to reduce separation requirements for future air transportation systems using airborne Automatic Dependent Surveillance-Broadcast (ADS-B) and Airborne Separation Assistance Systems (ASAS). EOO research was specifically focused on developing a globally accepted, early application of airborne ADS$B$ that results in more efficient oceanic and remote non-radar operations while providing opportunities for research of ASAS. This early application should provide operational expertise with ASAS and be an incentive for operators to voluntarily upgrade fleet equipment enabling system-wide transformation.

\section{In-Trail Procedure}

The ITP is described in the Operational Services and Environment Description (OSED) document [1] from the RTCA/EUROCAE Requirements Focus Group (RFG). The procedure requires the crew to use ADS$B$ information derived onboard the aircraft to determine if criteria required for an ITP are met. The aircraft-derived information includes flight identifier (ID), flight level, same direction of travel, ITP distance, and ground speed differential (all relative to potentially blocking aircraft). The equipment onboard the ITP enabled aircraft receives the ADS-B data and, along with onboard navigation data, calculates appropriate separation information for these aircraft and portrays this information to the crew. Using this information, the crew determines whether to request a standard or ITP flight level change. If the desired flight level appears available but potentially blocking aircraft are observed at the intervening flight levels and the requested flight level is no more than 4,000 feet (ft.) from the initial flight level, then the crew would evaluate the available information for these potentially blocking aircraft to determine if they can be used as reference aircraft in an ITP flight level change request. An aircraft at an intervening flight level can be used as a reference aircraft if it meets the following criteria:

- Same direction of flight as the ITP Aircraft, +/- 45 degrees,

- Qualified ADS-B data are being received from the aircraft, and

- $\quad$ ITP distance/speed criteria are met.

The equipment onboard the ITP enabled aircraft calculates for each aircraft the ITP distance, which is the difference in distance to a common point as shown in Figure 2 (for identical ground tracks, ITP distance is simply the distance between the two aircraft). The ITP speed criterion is a ground speed differential, also calculated by the equipment onboard the ITP enabled aircraft and is simply the difference in ground speed between the two aircraft. A positive ground speed differential is one which results in a decreasing ITP distance between the respective aircraft.

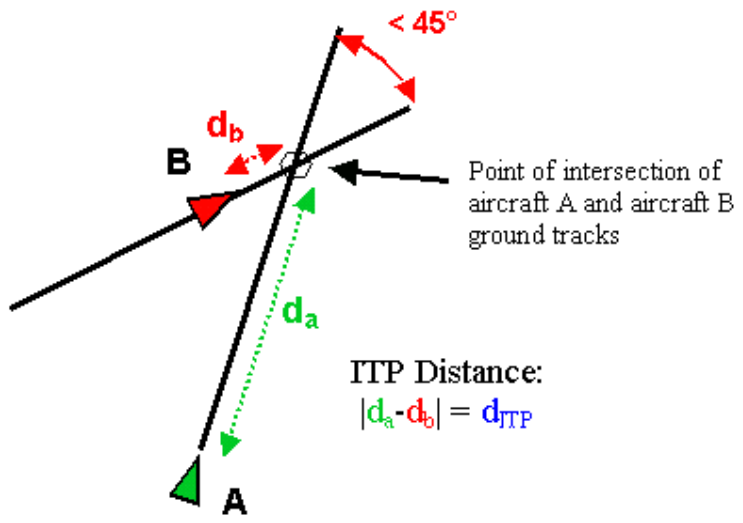

Figure 2. ITP distance

A reference aircraft must meet the following ITP distance/speed criteria, which were established during safety analyses of the procedure:

- ITP Distance at least 15 nautical miles (nmi), and positive ground speed differential of 20 knots (kts) or less, or

- ITP Distance at least $20 \mathrm{nmi}$, and positive ground speed differential of $30 \mathrm{kts}$ or less.

Up to two reference aircraft can be included in an ITP flight level change request to ATSP. All possible ITP geometries are permissible as long as all aircraft meet the criteria for reference aircraft.

In the simulation study an ITP flight level change was requested through a simulated data-link interface, similar to that required to make a standard flight level change request, with additional ITPspecific information entered on the available free text lines. The keyword "ITP" had to start the first (and only the first) free text line followed by the information of each of the one or two reference aircraft, according to the following format:

$\mathrm{F} /<$ reference aircraft flight id $>/ \mathrm{nn}$ or
$\mathrm{L} /<$ reference aircraft flight id $>/ \mathrm{nn}$

Where:

$\mathrm{F} /$ means following this reference aircraft

$\mathrm{L}$ / means leading this reference aircraft

$/ \mathrm{nn}$ is the ITP Distance, in nmi 
Given that the ITP is an airborne traffic situation awareness application there is no change in the responsibilities of either pilots or controllers. The flight crew continues to be responsible for the operation of the aircraft and conformance to clearances, and the controller continues to be responsible for separation and the issuance of clearances. However, the controller would use additional information transmitted in the ITP aircraft's request to determine if separation can be assured and a clearance can be issued.

Upon receipt of an ITP request, ATC would determine if a standard (non-ITP) flight level change clearance could be issued. If a non-ITP clearance is not possible, the controller would verify that the reference aircraft have not been cleared to change speed or flight level, are not about to reach a point at which a significant change of track will occur, and have a positive Mach difference of no greater than 0.04 relative to the requesting aircraft ${ }^{6}$. If these ITP ATC criteria are met and separation exists at the requested flight level with other aircraft, ATC may issue the ITP flight level change clearance.

If an ITP clearance is received, then the crew must reassess the reference aircraft identified in the clearance to assure that the ITP distance/speed criteria are still met before accepting the clearance. If the criteria are no longer met, then the clearance must be declined.

Once an ITP clearance has been accepted, the crew should commence the flight level change without delay and maintain cruise Mach number and at least 300 feet per minute (FPM) vertical speed throughout the flight level change. If this minimum performance cannot be maintained, then the applicable ICAO defined regional contingency procedures for inability to conform to an ATC clearance must be followed.

The ITP aircraft crew is not required to monitor the ITP distance to the reference aircraft during the climb or descent. The separation assurance of the ITP is ensured by the proper initial conditions and by maintenance of the minimum required vertical speed during the maneuver. The ITP is completed when the ITP flight crew reports being established at the new flight level.

${ }^{6}$ ATC checks the difference in (airspeed-based) assigned Mach numbers rather than groundspeed differential in knots as checked by the flight crew. The Mach check is required by the safety case to account for non-uniform winds at different flight levels.
Study Objectives and Hypotheses

The first objective of the EOO Human-In-The-Loop ITP Validation Simulation Study was to assess the validity of the ITP by determining if subject pilots: a) were able to perform ITP maneuvers during simulated oceanic flights, and b) found the procedural steps that they were instructed to use while executing ITP maneuvers to be correct, complete, and appropriately specified.

The second objective was to assess pilot acceptability of the ITP. In addition to collecting subject pilots' impressions of the overall acceptability of the ITP, the study attempted to determine if subject pilots found that: a) the level of workload that they experienced while performing ITP maneuvers was acceptable, and b) the ITP will likely provide perceived benefits possibly in the form of improved traffic situation awareness, smoother rides and/or fuel savings.

It was hypothesized that subject pilots would be able to perform ITP maneuvers (i.e., that the instructed procedural steps would be performed correctly and in the appropriate order) and that subject pilots would not find any missing, incomplete, or extraneous procedural steps associated with the ITP. Additionally, it was hypothesized that subject pilots would find the workload level associated with performing ITP flight level changes to be acceptable; that is, subject pilots were expected to provide workload ratings of " 3 " or less using the Modified Cooper-Harper (MCH) Rating Scale which ranges from 1 to 10 (1 = Minimal Operator Effort, $3=$ Medium Operator Effort, 7 = Maximum Operator Effort, and 10 = Task can not be accomplished) [2].

Note that this research endeavor only studied ITP validity and acceptability to pilots. Validity and acceptability to controllers were not considered in this experiment.

\section{Participants}

Participants consisted of 12 commercial airline pilots with current oceanic experience. All of the subject pilots were male and ranged in age between $42-59$ years [Mean $(M)=49$, Standard Deviation $(S D)=6$ ]. Five of the participants were captains, and the other seven were first officers. On average, the participants had 18 years of airline experience $(M=18.6, S D=$ 7.2) and over 9,000 hours of airline flying experience $(M=9,892, S D=5,829)$. All of the participants had experience with data-link communications and Automatic Dependent Surveillance-Contract (ADS- 
C). Two of the participants had previous experience using Electronic Flight Bag (EFB) devices, and one participant had previous experience using a Vertical Situation Display (VSD).

Test Facilities and Apparatus

The aircraft simulation used for this experiment, known as the NASA LaRC Aircraft Simulation for Traffic Operations Research (ASTOR) [3], is a medium-fidelity computer workstation-based desktop flight simulator whose displays and control panels are representative of a current-generation generic transport aircraft flight deck (e.g., a B-777).

In addition to the main ASTOR displays and control panels, the pilot interface for the experiment also included an EFB simulation. This EFB simulation hosted the ITP application and was run on a separate tablet personal computer (PC) to the left of the two ASTOR screens (see Figure 3). Interaction with the ITP application was accomplished by the subject pilots using a separate computer mouse from the one used to interact with the main ASTOR displays and control panels.

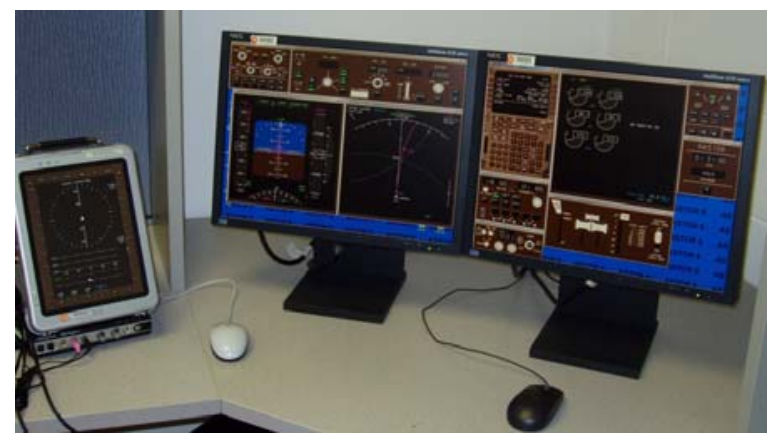

Figure 3. ASTOR layout

The ITP application consisted of a newly designed interface and a prototype implementation of the logic contained in the OSED document describing the ITP maneuver constraints. This ITP algorithm logic, described in a formal specification language [4], was used to determine the status of many of the elements on the ITP interface display (Figure 4Figure-4).

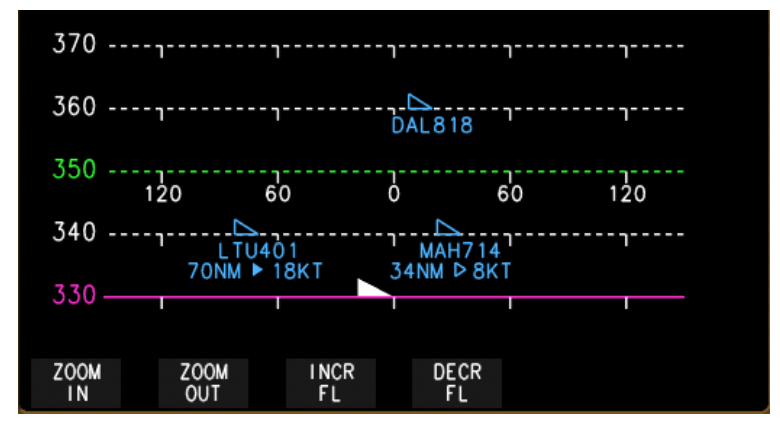

Figure 4. ITP application symbology

A desktop flight simulation program called the Traffic Manager (TMX) performed the function of background traffic generator and ATC simulation. TMX was originally developed by the National Aerospace Laboratory (NLR) in The Netherlands, and it can also serve as a stand-alone traffic simulator, flight simulation scenario generator and editor, experiment control station, data-recording tool, and rapid prototyping environment [5].

\section{Experiment Design}

The experiment design used for data collection was an 8 (Type of maneuver) x 2 (Ability to complete maneuver) full-factorial, within-subject design (Table $1)$. The twelve subject pilots $\left(S_{1-12}\right)$ performed all 16 test conditions in random order.

Table 1. Experiment Design Matrix

\begin{tabular}{|l|l|l|}
\hline Type of maneuver & $\begin{array}{l}\text { Able to } \\
\text { complete }\end{array}$ & $\begin{array}{l}\text { Not able to } \\
\text { complete }\end{array}$ \\
\hline Standard Climb & $\mathrm{S}_{1-12}$ & $\mathrm{~S}_{1-12}$ \\
\hline Standard Descent & $\mathrm{S}_{1-12}$ & $\mathrm{~S}_{1-12}$ \\
\hline ITP Following Climb & $\mathrm{S}_{1-12}$ & $\mathrm{~S}_{1-12}$ \\
\hline ITP Following Descent & $\mathrm{S}_{1-12}$ & $\mathrm{~S}_{1-12}$ \\
\hline ITP Leading Climb & $\mathrm{S}_{1-12}$ & $\mathrm{~S}_{1-12}$ \\
\hline ITP Leading Descent & $\mathrm{S}_{1-12}$ & $\mathrm{~S}_{1-12}$ \\
\hline ITP Combined Climb & $\mathrm{S}_{1-12}$ & $\mathrm{~S}_{1-12}$ \\
\hline ITP Combined Descent & $\mathrm{S}_{1-12}$ & $\mathrm{~S}_{1-12}$ \\
\hline
\end{tabular}

Note: The reference aircraft is located behind the ITP aircraft during a leading maneuver, and ahead of the ITP aircraft during a following maneuver.

A flight level change was considered "Not able to complete" if either the target and/or intermediate flight level was blocked by traffic aircraft of which the subject pilot was unaware (i.e., controller rejects request) or blocked by traffic aircraft of which the subject pilot was aware (i.e., pilot should not request flight level change).

The dependent measures for the experiment included selection errors, execution errors and subjective assessments of the ITP's validity, acceptability and 
workload. Selection errors occurred when subject pilots made a flight level change request that was different from the expected request for a given scenario. Execution errors occurred if subject pilots: a) failed to correctly communicate required information to ATC while making either a standard or an ITP flight level change request, or b) failed to adhere to the aircraft performance criteria required during ITP flight level change maneuvers. Depending on the potential consequences associated with a given execution error, it may have been identified as a "safety-related execution error."

The subject pilots' perceptions regarding the validity, acceptability and workload of the ITP were collected using: 1) a post-scenario questionnaire that was administered after the completion of each test condition, 2) a post-experiment questionnaire that was administered after the completion of the final test condition, and 3) a post-experiment group debrief session.

\section{Results}

Subject pilots participated in the study in groups of three to six, with each group participating in the study over the course of two consecutive days. Approximately the first half day consisted of a combination of "classroom" and hands-on ITP and experiment procedures training. Collectively, the subject pilots performed 192 simulated flight scenarios while completing the experiment's 16 test conditions. The 192 simulated flight scenarios consisted of 48 (4 standard scenarios x 12 subject pilots) standard flight level changes and 144 (12 ITP scenarios x 12 subject pilots) ITP flight level changes.

\section{Selection Errors}

The subject pilots made 19 selection errors during the 192 scenarios, yielding an overall selection error rate of $9.9 \%$. During the 48 expected standard scenarios, subject pilots made a total of six ITP flight level change requests. That is, $12.5 \%$ of the time subject pilots requested an ITP flight level change when they were expected to request a standard flight level change. However, the subject pilots may have been biased towards making ITP requests since they were instructed during their training: "when in doubt [about aircraft spacing], request an ITP.”

Thirteen selection errors occurred during the 144 expected ITP scenarios resulting in an ITP selection error rate of $9.0 \%$. Four of the 13 ITP selection errors involved instances in which subject pilots requested an ITP flight level change when they were expected to have realized that an ITP maneuver was not possible because an observable traffic aircraft was blocking the ITP maneuver. Seven ITP selection errors occurred when subject pilots included an additional reference aircraft (located at the desired altitude) in their request for an expected leading or following ITP scenario. One ITP selection error involved a subject pilot failing to reference both the leading and following aircraft in what was expected to be a combined ITP flight level change. The remaining ITP selection error involved a subject pilot requesting a standard flight level change when he was expected to determine that a standard flight level change was not possible due to blocking aircraft, nor was an ITP maneuver possible because the ground speed differential of the blocking aircraft did not meet the required ITP speed criteria for a reference aircraft.

\section{Execution Errors}

Execution errors were evaluated after subject pilots chose to request a given type of flight level change maneuver. As a result of the subject pilots' selection errors, there was a change in the total number of standard scenarios and ITP scenarios during which execution errors could occur. Of the original 48 expected standard scenarios, six were "converted into" ITP scenarios, and one ITP selection error resulted in the conversion of an expected ITP scenario into a standard scenario. Therefore, subject pilots requested a total of 43 standard flight level changes and 149 ITP flight level changes (Table 2).

Table 2. Expected versus Requested Scenarios

\begin{tabular}{|l|l|l|l|l|}
\hline & \multicolumn{2}{|l|}{ Standard } & \multicolumn{2}{l|}{ ITP } \\
\cline { 2 - 5 } & Expected & Requested & Expected & Requested \\
\hline Total & 48 & 43 & 144 & 149 \\
\hline
\end{tabular}

Subject pilots did not commit any execution errors during the 43 requested standard scenarios.

During 29 of the 149 (19.5\%) requested ITP scenarios, subject pilots committed at least one execution error. Since it was possible for a subject pilot to make multiple errors during a single scenario, a total execution error count of 33 occurred. Of the total execution error count, nine were identified as involving safety-related execution errors. In general, two types of errors were classified as being safetyrelated: 1) failure to adhere to performance requirements, and 2) the inclusion of inaccurate information in a flight level change request that could either not be verified by ATC or not be evaluated by ATC as containing inaccuracies. 
Two of the safety-related ITP execution errors occurred when subject pilots identified a leading aircraft as a following aircraft. Two safety-related ITP execution errors occurred when subject pilots failed to include the third number of a reference aircraft's call sign, and three safety-related ITP execution errors occurred when subject pilots referenced an incorrect aircraft. The remaining two safety-related ITP execution errors occurred when one subject pilot failed, in two separate scenarios, to adhere to the aircraft performance criteria required during a requested ITP scenario. In one instance, the subject pilot made a deliberate speed change of 0.01 Mach that allowed him to perform a maneuver that otherwise would not have been allowed; in the other instance, the same subject pilot failed to maintain required vertical speed.

A McNemar Test (i.e., a nonparametric withinsubject test appropriate for analyzing two related samples of nominal data) [6] showed that significantly fewer safety-related execution errors occurred during the requested standard scenarios (i.e., no errors) than during the requested ITP scenarios ( $\mathrm{p}$ $<0.01)$.

Twenty-four "non-safety-related execution errors" occurred during the scenarios involving ITP flight level changes. Sixteen ITP execution errors involved instances in which subject pilots referenced an aircraft at the desired altitude during a flight level change request; three ITP execution errors involved subject pilots requesting a climb or descent to an incorrect altitude; three ITP execution errors were related to syntax errors in the ITP phraseology; one ITP execution error occurred when a subject pilot failed to comply with the ATC instruction to "report reaching" when level at the completion of the approved maneuver (per ICAO procedures for oceanic/remote flight operations); and one ITP execution error involved a subject pilot failing to accept ATC's denial of an ITP flight level change request (per the briefed standard procedures for datalinked ATC communications) before making a second ITP flight level change request.

\section{Subjective Assessments of the ITP's Validity}

The subject pilots indicated in $92.7 \%$ of their postscenario questionnaire responses that the procedural steps used to execute ITP maneuvers were correct, complete, and appropriately specified. Suggestions were made to improve the checklist by including additional steps (i.e. confirmation steps between Pilot Flying and Pilot Not Flying) to prevent oversight and to avoid syntax errors. Two of the subject pilots indicated they used the ITP checklist during every ITP maneuver while, on average, the other 10 subject pilots reported using the checklist during 37\% (SD = $17.4 \%$ ) of the ITP maneuvers. When asked if they reassessed the ITP criteria every time they received an ITP clearance, as required by the checklist, eight of the subject pilots stated that they always performed the required reassessment while four of the subject pilots stated that they did not adhere to this procedural step. When asked to describe their ITP performance concerns, the subject pilots mentioned the existence of wake vortices during the execution of an ITP maneuver; however, it was acknowledged that the impact of wake vortices must also be considered during current day operations. Second, the potential consequences associated with failures of aircraft to comply with ITP performance requirements were identified as a cause for concern.

\section{Subjective Assessments of the ITP’s Acceptability}

All 12 subject pilots found the ITP to be an acceptable procedure, but two subject pilots mentioned concerns regarding the procedure's reliance on an "honor system" in which the distances among aircraft must be accurately reported to ATC. Two subject pilots indicated that the ITP's acceptability would increase if pilots were afforded the opportunity to communicate alternate altitude requests to ATC in hopes of securing approval for a flight level change should their initial requests be denied. With respect to their impressions regarding the level of safety associated with performing the ITP as compared with current day procedures, one subject pilot characterized the ITP as being "less safe than" current day procedures but stated that the ITP is still "procedurally sound"; seven subject pilots characterized the ITP as being "equally as safe as" current day procedures; and four subject pilot characterized the ITP as being "safer than" current day procedures. Subject pilots indicated that the improved safety was a direct result of the enhanced situation awareness (SA) provided by the ITP application's display. When asked to comment on their use of free text phraseology to communicate with "ATC," subject pilots indicated that, in general, they found the terminology to be straightforward and easy to learn and felt that "Leading" and "Following" were good terms to use since it is logical for pilots to reference their ITP Aircraft. It was pointed out, however, that airlines often discourage the use of free text due to language barriers and the possibility of misinterpretation. Therefore, one subject pilot suggested that the ITP display provide "clickable" information that could be used to populate free text fields to reduce typographical errors and/or language 
differences (e.g., ITP climb/descend could be selectable). When asked about potential benefits that the ITP might have, the subject pilots identified: improved traffic SA; more efficient aircraft operation (resulting from the ability to manage climbs at efficient times); enhanced job satisfaction associated with the opportunity to achieve optimum performance; and improvements in safety due to the avoidance of weather and turbulence.

In addition to providing feedback regarding the ITP's overall acceptability, safety, and phraseology, the subject pilots also shared enlightening viewpoints regarding ATC's role in separation assurance. Several of the subject pilots indicated that if the appropriate information is made available to flight crews, then the level of responsibility for separation assurance that is given to pilots during ITP maneuvers is acceptable. However, when the potential for increasing pilot responsibility for separation assurance was considered, one commonly expressed opinion was that pilots do not want more responsibility for separation assurance, particularly because of "violation potential," and that pilots have "no interest in being an air traffic controller."

\section{Subjective Assessments of the ITP's Workload}

After completing each of the experiment's 16 test conditions, subject pilots used the MCH Rating Scale to report the level of workload that they had just experienced.

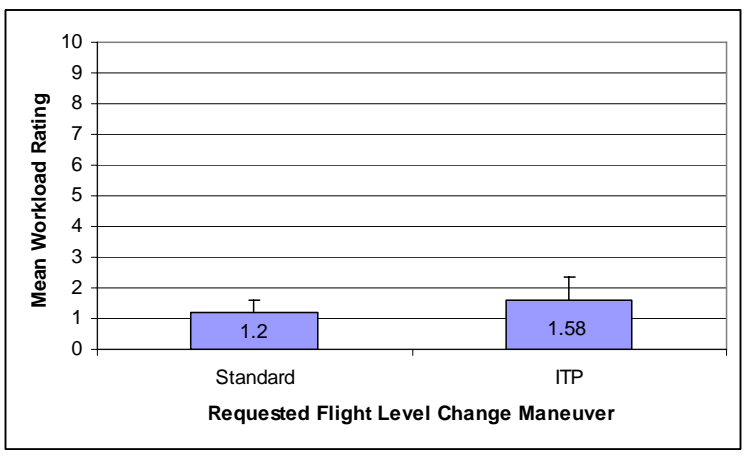

Figure 5. Mean MCH workload ratings

The MCH workload ratings associated with the requested standard scenarios revealed a mean of 1.20 [SD $=0.40$, Sample Size $N=43]$, and the $\mathrm{MCH}$ workload ratings associated with the requested ITP scenarios revealed a mean of $1.58(S D=0.75, N=$ 149) (Figure 5Figure 5). A Wilcoxon Test (i.e., a nonparametric within-subject test appropriate for analyzing two related samples of ordinal data) [7] revealed that a significantly higher workload rating was associated with the requested ITP flight level change maneuvers compared to requested standard flight level change maneuvers ( $p<0.01)$. It is asserted, however, that the difference is not operationally significant. Subject pilots indicated that other phases of flight (i.e. descent and approach) have much higher workload ratings than ITP flight level change maneuvers.

\section{Discussion}

When the overall results are examined, it is apparent that in the majority of scenarios, subject pilots were able to correctly assess the traffic situation, select an appropriate response, and execute their selected flight level change procedure, if any, without error. However, it could also be asserted that the error rates for ITP maneuvers were higher than for standard flight level changes, and that these errors have design implications for both the ITP and the prototype traffic display. Nine of the 33 execution errors were specified as "safety-related" (errors that potentially cannot be detected by ATC). These errors, however, might be mitigated by: 1) improving the prototype ITP operation's display interface and symbology; 2) limiting the applicability of the ITP to well-defined simple geometries; 3) defining specific rules-ofthumb (versus the current judgment-based guidance) as to when pilots should request an ITP; and/or 4) increasing the level of automation/decision support provided by the ITP operations application.

There was also an instance where the subject pilot "gamed" the scenario by intentionally changing his speed by 0.01 Mach to allow an ITP that would not otherwise have been possible. The possibility of pilots "gaming” such situations was widely discussed during the debrief sessions with the subject pilots, and the pilots' consensus was that such behavior would inevitably occur occasionally, at some unknown frequency, but would not be considered hazardous to safety. Note, however, that oceanic clearances typically require that speed be maintained to within 0.01 Mach to maintain longitudinal separation in non-radar environments.

Sixteen of the 24 non-safety-related execution errors (i.e., two-thirds of these errors) involved subject pilots incorrectly referencing aircraft at the desired final, rather than the intervening, flight level. Why the above-described errors occurred, and especially why they occurred in such large numbers, is a source of significant puzzlement and speculation among the authors. During the debrief sessions subject pilots were asked for possible explanations. The answers varied from: either the subject pilots didn't realize 
they had made the error and couldn't say why they had done so; they did it by mistake and recognized afterward that they had done so; or they intentionally did it to let ATC know that, for example, they "knew about the presence of that aircraft.”

\section{Conclusions}

Analysis of both the qualitative and quantitative data revealed that the ITP, from a pilot's perspective, is generally both valid and acceptable within the conditions of the experiment. Although the workload level reported for the ITP flight level change requests was higher than the workload level reported for the standard flight level change requests, subjective workload assessments were well within acceptable limits. In addition, the subject pilots' subjective acceptability ratings and comments regarding the ITP were generally high and positive, respectively. While the ITP was generally regarded to be valid and acceptable, the error rates for ITP maneuvers were higher than for standard flight level changes, but mitigating strategies were suggested.

In the end, the subject pilots found the procedure valid, acceptable, "procedurally sound," and "intuitively obvious" and were very enthusiastic about the enhanced SA provided by the ITP operations application display. The subject pilots were somewhat unenthusiastic regarding the notion of more pilot responsibility for separation assurance in future applications, but would like to see the presented ITP implemented during current day oceanic flight operations.

\section{Acknowledgements}

The authors gratefully acknowledge the significant contributions of the many members of the research team from both Government and Industry that were required to conduct this experiment. Deserving of credit are Tom Graff of the National Institute of Aerospace, Terry Abbott of Booz-Allen \& Hamilton, and Victor Carreno of the National Institute of Aerospace, as well as contractor counterparts from Raytheon, SAIC, Lockheed-Martin, Sensis, and the National Institute of Aerospace. Many other persons whose names are not mentioned here have contributed significantly, and the authors express their sincerest thanks to them as well.

\section{References}

[1]

[2]

[3]
OSED In-Trail Procedure in Non-Radar

Oceanic Airspace, version 5.0,

RTCA/EUROCAE Requirements Focus Group, Application Definition Sub-group, working draft.

Wierwille, W. W.; and Casali, J. G.: A Valid

Rating Scale for Global Mental Workload

Measurement. Proceedings of the Human Factors Society $27^{\text {th }}$ Annual Meeting, 1983, pp. 129-133.

Peters, Mark E., Ballin, Mark G., \& Sakosky, John S. (2002). A Multi-Operator Simulation for Investigation of Distributed Air Traffic Management Concepts. AIAA Paper 2002-4596. Reston, VA: American Institute of Aeronautics and Astronautics. César Munoz, Radu Siminiceanu, In-Trail Procedure (ITP) Algorithm Design, NIA Report No. 2007-06, National Institute of Aerospace, Hampton, VA.

Bussink, F.J.L., Hoekstra, J., Heesbeen, W., Traffic Manager: A Flexible Desktop Simulation Tool Enabling Future ATM Research, 24 ${ }^{\text {th }}$ Digital Avionics Systems Conference, October 2005, Washington DC. Norusis, M. J.: SPSS ${ }^{\circledR}$ for Windows ${ }^{\mathrm{TM}}$ : Base System User's Guide, Release 6.0. SPSS Inc. (Chicago, IL), 1993.

Winer, B. J.; Brown, D. R.; and Michels, K. M.: Statistical Principles in Experimental Design. Third ed., McGraw-Hill, Inc., 1991. 